\title{
Constructive Interference for Generic Constellations
}

\author{
Alireza Haqiqatnejad, Student Member, IEEE, Farbod Kayhan, and Björn Ottersten, Fellow, IEEE
}

\begin{abstract}
In this letter, we investigate optimal and relaxed constructive interference regions (CIR) for the symbol-level precoding (SLP) problem in the downlink of a multiuser multipleinput single-output (MISO) channel. We define two types of CIRs, namely, distance preserving CIR (DPCIR) and union bound CIR (UBCIR) for any given constellation shape and size. We then provide a systematic way to describe these regions as convex sets. Using the definitions of DPCIR and UBCIR, we show that the SLP power minimization problem, minimizing either sum or peak (per-antenna) transmit power, can always be formulated as a convex optimization problem. Our results indicate that these regions allow further reduction of the transmit power compared to the current state of the art without increasing the computational complexity at the transmitter or receiver.
\end{abstract}

Index Terms-Constructive interference, multiuser MISO, symbol-level precoding, Voronoi regions.

\section{INTRODUCTION}

Transmit beamforming in the downlink of multiuser multiple-antenna channels, which is commonly referred to as multiuser precoding, has been widely studied in the literature (see for example [1] and the references within). In general, this problem is formulated as a constrained optimization problem, where the objective function and the constraints are defined so as to keep a balance between the available resources and the target quality-of-service (QoS). Some well-known formulations for this problem are power minimization with signalto-interference-plus-noise ratio (SINR) constraints, max-min SINR balancing, and (weighted) sum-rate maximization [2] [3]. In the power minimization problem with SINR constraints, which is the main focus of this letter, the goal is to minimize the sum or peak (per-antenna) transmit power subject to individual SINR requirements.

Conventional multiuser precoding schemes try to design the precoder in order to mitigate the multiuser interference. For quasi-static fading scenarios, the precoder is redesigned in each block using the instantaneous channel state information (CSI). These methods can be interpreted as block-level precoding. On the other hand, symbol-level precoding (SLP) tries to turn the interference into a useful source of desired signal power instead of considering it as an unwanted distortion to be mitigated or eliminated. This leads to introducing the notion of constructive interference (CI), which was first presented in [4]. Accordingly, in addition to CSI, the instantaneous data information (DI) of all users are used to design the precoder. An SLP optimization problem then needs to be solved for each symbol period. It has been shown that significant gains can be achieved with respect to conventional schemes at the cost of higher transmitter complexity [5] [6]. One can also formulate

The authors are with Interdisciplinary Centre for Security, Reliability and Trust (SnT), University of Luxembourg, L-1855 Luxembourg. (email: \{alireza.haqiqatnejad,farbod.kayhan,bjorn.ottersten\}@uni.lu). the SLP problem to directly find the optimal transmit vector, as proposed in [6], instead of finding the precoding matrix.

The constraints in the SLP optimization problem push each noiseless received signal to its correct constructive interference region (CIR), and therefore, the constraints depend on the constellation shape. In [5] and [6], the authors formulate the SLP optimization problem only for PSK constellations, considering sum transmit power, and the extension to peak per-antenna power is presented in [7]. The problem is also addressed for QAM and APSK constellations in [8] and [9], respectively. A generic formulation (i.e., not depending on a specific constellation) for the SLP optimization problem is not provided in the literature.

In this letter, we first discuss optimal and relaxed CIRs for a generic constellation, and then show that the SLP problem can always be formulated as a convex optimization problem defined on these regions. We provide explicit convex formulations for the SLP power minimization problem, and compare their performances and computational complexities with the state of the art techniques.

The rest of this letter is organized as follows. In Section II we describe our system model and review the CI-based SLP problem. We discuss optimal and relaxed CIRs for a generic constellation in Section III. In Section IV we formulate the SLP power minimization problem as a convex optimization problem for given CIRs. We present the simulation results in Section V. Finally, we conclude the letter in Section VI.

\section{System Model And Problem Definition}

We consider the downlink of a multiuser multiple-input single-output (MISO) channel where a single base station (BS) with $N$ transmit antennas simultaneously serves $K$ users, each equipped with a single receive antenna. A block fading channel is assumed between any transmit/receive antenna pair. We denote by $\mathbf{h}_{k} \in \mathbb{C}^{1 \times N}$ the channel vector between the BS transmit antennas and the $k$-th user, and by $\mathbf{H}=$ $\left[\mathbf{h}_{1}^{T}, \ldots, \mathbf{h}_{K}^{T}\right]^{T}$ the channel matrix, where $[\cdot]^{T}$ denotes the transpose operator. Throughout the letter, we drop the symbol's time index to simplify the notation. At a given symbol period, $K$ independent data symbols are collected in users' symbol vector $\mathbf{s}=\left[s_{1}, \ldots, s_{K}\right]^{T} \in \mathbb{C}^{K \times 1}$ with $s_{k}$ denoting the symbol intended for the $k$-th user. The elements of $\mathbf{s}$ are drawn from (potentially non-identical) finite equiprobable twodimensional constellation sets. Without loss of generality, we assume a generic constellation set $\chi=\left\{x_{i} \mid x_{i} \in \mathbb{C}\right\}_{i=1}^{M}$ for all users, where $M$ is the modulation order. We further assume that $\chi$ has unit average power, i.e., $\mathbb{E}_{\chi}\left\{\left|x_{i}\right|^{2}\right\}=1$.

Assuming perfect channel knowledge, the user's symbol vector is mapped to $N$ transmit antennas by a symbol-level precoder resulting in the signal vector $\mathbf{u} \in \mathbb{C}^{N \times 1}$ to be 
transmitted by the BS. The received signal by the $k$-th user is then $r_{k}=\mathbf{h}_{k} \mathbf{u}+w_{k}$, where $w_{k} \sim \mathcal{C N}\left(0, \sigma_{k}^{2}\right)$ is the complex additive white Gaussian noise (AWGN) at the $k$ th receiver. Again without loss of generality, we assume identical noise distributions at the receiver of different users, i.e., $\sigma_{k}=\sigma, k=1, \ldots, K$. From the received scalar $r_{k}$, the user $k$ tries to detect its own symbol using the maximum-likelihood (ML) decision rule.

An effective measure of QoS in multiuser interference channels is SINR [10], which is translated to received SNR when $\mathrm{CI}$ is of interest. In the SLP power minimization problem, the transmit vector $\mathbf{u}$ is designed instantaneously in each symbol period based on a constrained optimization problem, and is in general a function of $\mathbf{s}, \mathbf{H}$, and the set of given SNR thresholds for all users. The SNR-related constraints can be expressed as

$$
\left|\mathbf{h}_{k} \mathbf{u}\right|^{2} \geq \sigma^{2} \gamma_{k}\left|s_{k}\right|^{2}, k=1, \ldots, K,
$$

where $\gamma_{k}$ is the SNR threshold for the $k$-th user. Notice that the SNR thresholds $\left\{\gamma_{k}\right\}_{k=1}^{K}$ typically refer to long-term (e.g., frame-level) SNRs, i.e., the average received SNR over all the symbols in a frame. For sufficiently large frames we have $\mathbb{E}\left\{\left|s_{k}\right|^{2}\right\} \rightarrow 1, k=1, \ldots, K$, and therefore the symbollevel constraints (1) satisfy the frame-level SNR thresholds $\mathbb{E}\left\{\left|\mathbf{h}_{k} \mathbf{u}\right|^{2}\right\} \geq \sigma^{2} \gamma_{k}, k=1, \ldots, K$, where the expectations are taken over the entire frame. In this letter, we use the symbollevel SNR constraints (1), although they may not be in general necessary conditions to meet the frame-level SNR thresholds.

Considering (1), the SLP power minimization problem has been formulated in [5] and [6] as

$$
\begin{aligned}
\mathbf{u}(\mathbf{s}, \mathbf{H}, \boldsymbol{\gamma})=\underset{\mathbf{u}}{\operatorname{argmin}} & f(\mathbf{u}) \\
\text { s.t. } & \mathbf{h}_{k} \mathbf{u} \in \sigma \sqrt{\gamma_{k}} \mathcal{D}_{k}, k=1, \ldots, K,
\end{aligned}
$$

where $\mathcal{D}_{k}$ represents the CIR associated with symbol $s_{k}, \gamma$ is the vector collecting all the SNR thresholds $\left\{\gamma_{k}\right\}_{k=1}^{K}$, and the objective function $f(\mathbf{u})$ can be either $\|\mathbf{u}\|_{2}^{2}$ or $\|\mathbf{u}\|_{\infty}^{2}$ depending on whether sum or peak (per-antenna) transmit power is minimized. The constraints in (2) satisfy the SNR constraints (1) if the amplitude of any point in $\mathcal{D}_{k}$ is equal to or larger than $\left|s_{k}\right|$, for all $k=1, \ldots, K$. We call this the amplitude condition in what follows.

\section{Constructive Interference Regions}

In this section, we define several types of CIRs and describe them in a systematic way based on the ML decision regions of the constellation $\chi$. Hereinafter, we denote each complexvalued constellation point by its equivalent real-valued vector form, and thus the set of points in $\chi$ is denoted by $\left\{\mathbf{x}_{i} \mid \mathbf{x}_{i} \in\right.$ $\left.\mathbb{R}^{2}\right\}_{i=1}^{M}$.

The ML decision rule for the constellation set $\chi$ has a geometric interpretation; it corresponds to the Voronoi regions of $\chi$ which are bounded by hyperplanes. Assuming a given constellation point $\mathbf{x}_{i}$ and one of its neighboring points $\mathbf{x}_{j}$, the hyperplane separating the Voronoi region of $\mathbf{x}_{i}$ from that of $\mathbf{x}_{j}$ is described as $\left\{\mathbf{x} \mid \mathbf{x} \in \mathbb{R}^{2}, \mathbf{a}_{i, j}^{T} \mathbf{x}=b_{i, j}\right\}$, where $\mathbf{a}_{i, j} \in \mathbb{R}^{2}$, $\mathbf{a}_{i, j} \neq \mathbf{0}$, and $b_{i, j} \in \mathbb{R}$. This hyperplane is a decision boundary between $\mathbf{x}_{i}$ and $\mathbf{x}_{j}$, which splits $\mathbb{R}^{2}$ into two halfspaces. The halfspace extending towards $\mathbf{x}_{i}$ is the solution set of a linear inequality represented by $\left\{\mathbf{x} \mid \mathbf{x} \in \mathbb{R}^{2}, \mathbf{a}_{i, j}^{T} \mathbf{x} \geq b_{i, j}\right\}$, where the vector $\mathbf{a}_{i, j}$ is the inward normal of this halfspace, and the constant $b_{i, j}$ determines the offset from the origin. The Voronoi region of $\mathbf{x}_{i}$ is then given by intersecting all such halfspaces, i.e.,

$$
\mathcal{D}_{i, \mathrm{ML}}=\left\{\mathbf{x} \mid \mathbf{x} \in \mathbb{R}^{2}, \mathbf{a}_{i, j}^{T} \mathbf{x} \geq b_{i, j}, \forall \mathbf{x}_{j} \in \mathcal{S}_{i}\right\}
$$

where $\mathcal{S}_{i}$ denotes the set of points sharing a decision boundary with $\mathbf{x}_{i}$, and cardinality $\left|\mathcal{S}_{i}\right|=M_{i}$. Equation (3) can be written in a more compact form as

$$
\mathcal{D}_{i, \mathrm{ML}}=\left\{\mathbf{x} \mid \mathbf{x} \in \mathbb{R}^{2}, \mathbf{A}_{i} \mathbf{x} \succeq \mathbf{b}_{i}\right\}
$$

where $\mathbf{A}_{i} \in \mathbb{R}^{M_{i} \times 2}$ and $\mathbf{b}_{i} \in \mathbb{R}^{M_{i}}$ contain $\mathbf{a}_{i, j}^{T}$ and $b_{i, j}$, respectively, for all $\mathbf{x}_{j} \in \mathcal{S}_{i}$, and $\succeq$ denotes the componentwise inequality. The Voronoi region (4) can be either an unbounded polyhedron, if $\mathbf{x}_{i}$ is an outer constellation point, or a bounded polyhedron (polytope), if $\mathbf{x}_{i}$ is an inner point. It is easy to show that polyhedra, and hence the Voronoi regions, are always convex sets [11].

Each normal vector $\mathbf{a}_{i, j}$ in (3) is orthogonal to the Voronoi edge shared by $\mathbf{x}_{i}$ and $\mathbf{x}_{j}$, thus it can be obtained as $\mathbf{a}_{i, j}=$ $\mathbf{x}_{i}-\mathbf{x}_{j}$ (or any scalar multiplication of $\mathbf{x}_{i}-\mathbf{x}_{j}$ ). Furthermore, this Voronoi edge passes through the point $\left(\mathbf{x}_{i}+\mathbf{x}_{j}\right) / 2$, and therefore the corresponding offset $b_{i, j}$ in (3) is obtained by simple vector algebra as $b_{i, j}=\mathbf{a}_{i, j}^{T}\left(\mathbf{x}_{i}+\mathbf{x}_{j}\right) / 2$ [11, p. 27]. Notice that $b_{i, j}$ is found such that the orthogonal distance between $\mathbf{x}_{i}$ and the corresponding hyperplane is equal to half of the distance between $\mathbf{x}_{i}$ and $\mathbf{x}_{j}$. By changing $b_{i, j}$ to $b_{i, j}+$ $\delta_{i, j}$, where $\delta_{i, j} \geq 0$, we get a new hyperplane displaced by $\delta_{i, j} /\left\|\mathbf{a}_{i, j}\right\|_{2}$ in the direction of $\mathbf{a}_{i, j}$ (thus being parallel to the original hyperplane).

According to the definition of CI [5] [6], the CIR associated with $\mathbf{x}_{i}$ should be a subset of $\mathcal{D}_{i, \mathrm{ML}}$. In this letter we propose a construction method such that each CIR is obtained by displacement of the hyperplanes contributing to $\mathcal{D}_{i, \mathrm{ML}}$. The displacement values $\delta_{i, j}$ must be chosen carefully as they determine the margins from the Voronoi decision boundaries and thus affect the symbol error rate (SER). It is clear that for a fixed SNR, reducing the margins causes higher SER. On the other hand, from (2) it is deduced that for a given SNR threshold, having narrower margins gives larger search domain to find the vector $\mathbf{u}$ with minimum transmit power.

\section{A. Distance Preserving Constructive Interference Regions}

We call a CIR distance preserving (DPCIR) if it does not decrease the original distances between the constellation points. As a consequence, the achievable SER will be always lower than that of the original constellation. Let $d_{i, j}$ denote the distance between the points $\mathbf{x}_{i}$ and $\mathbf{x}_{j}$, the distance preserving margin is then equal to $d_{i, j} / 2$. Obtaining $\delta_{i, j}$ from $\delta_{i, j} /\left\|\mathbf{a}_{i, j}\right\|_{2}=d_{i, j} / 2$, the DPCIR associated with $\mathbf{x}_{i}$ is expressed as

$$
\mathcal{D}_{i, \mathrm{DP}} \stackrel{\text { def }}{=}\left\{\mathbf{x} \mid \mathbf{x} \in \mathbb{R}^{2}, \mathbf{A}_{i} \mathbf{x} \succeq \mathbf{b}_{i}+\Delta_{i, \mathrm{DP}}\right\},
$$

where $\Delta_{i, \mathrm{DP}} \in \mathbb{R}^{M_{i}}$ is the vector containing $d_{i, j}\left\|\mathbf{a}_{i, j}\right\|_{2} / 2$ for all $\mathbf{x}_{j} \in \mathcal{S}_{i}$. From (5) it follows that if $\mathbf{x}_{i}$ is an inner constellation point, $\mathcal{D}_{i, \mathrm{DP}}$ will be only $\mathbf{x}_{i}$ itself, and thus 
the noiseless received signal should exactly locate at $\mathbf{x}_{i}$. In case where $\mathbf{x}_{i}$ is an outer point, $\mathcal{D}_{i, \mathrm{DP}}$ will be a convex cone with a vertex at $\mathbf{x}_{i}$, and the noiseless received signal can lie anywhere on this cone. Figure 1 illustrates the DPCIRs (blue shaded regions with blue dotted boundary edges) for an 8-ary constellation which is optimized over AWGN channel using the method presented in [12]. Notice that if SER is not allowed to increase, then DPCIRs are optimal and correspond to the CIRs introduced for PSK and QAM constellations in [5] and [8], respectively.

\section{B. Union Bound Constructive Interference Regions}

In practice, the users may have some flexibility in terms of SER. In such cases, one may relax the DPCIRs as long as a given target SER is ensured. By doing so, we may have larger search domains available for the solution of the SLP problem (2), and possibly lower transmit powers are achievable. The relaxation can be done by bringing the CIR hyperplanes closer to the Voronoi decision boundaries.

In what follows, we use the union bound on symbol error probability to determine how close the CIR hyperplanes can get to the Voronoi boundaries. A tractable form of the union bound, known as the nearest neighbor union bound (NNUB) [13], is given by

$$
P_{\mathrm{e}, \mathrm{ML}} \leq\left(\frac{1}{M} \sum_{i} M_{i}\right) Q\left(\frac{d_{\mathrm{min}}}{2 \sigma}\right),
$$

where $Q(\cdot)$ is the standard Q-function and $d_{\min }$ is the minimum distance of the constellation. The NNUB gives a tight bound on symbol error probability being quite close to the exact SER at high SNRs. Notice that in our model, the received signal $r_{k}$ can be considered as the output of an AWGN channel and therefore the NNUB is valid.

Using (6), for a given $P_{\mathrm{e}}$, we define the distance threshold $d_{\text {min,UB }}$ as

$$
d_{\text {min, } \mathrm{UB}}=2 \sigma Q^{-1}\left(\frac{M P_{\mathrm{e}}}{\sum_{i} M_{i}}\right) .
$$

The value of $d_{\text {min,UB }}$ implies how far the noiseless received signal is allowed to deviate from the desired symbol without violating the target SER. Hence, $d_{\min , \mathrm{UB}}$ as defined in (7) is the smallest minimum distance by which the worst SER performance is guaranteed to be $P_{\mathrm{e}}$. This provides us with the intervals $\left[d_{\text {min, UB }}, d_{i, j}\right]$ from which we can choose the relaxed distances; however, the most energy-efficient choice is $d_{\text {min,UB }}$. We refer to these regions as union bound CIRs (UBCIR). Notice that in the general case of unequal target SERs, UBCIRs can be obtained separately for each user.

Obtaining the displacement values $\delta_{i, j}$ from $\delta_{i, j} /\left\|\mathbf{a}_{i, j}\right\|_{2}=$ $d_{\text {min, UB }} / 2$, the UBCIR associated with $\mathbf{x}_{i}$ is defined as

$$
\mathcal{D}_{i, \mathrm{UB}} \stackrel{\text { def }}{=}\left\{\mathbf{x} \mid \mathbf{x} \in \mathbb{R}^{2}, \mathbf{A}_{i} \mathbf{x} \succeq \mathbf{b}_{i}+\Delta_{i, \mathrm{UB}}\right\},
$$

where the vector $\Delta_{i, \mathrm{UB}} \in \mathbb{R}^{M_{i}}$ contains $d_{\text {min, } \mathrm{UB}}\left\|\mathbf{a}_{i, j}\right\|_{2} / 2$ for all $\mathbf{x}_{j} \in \mathcal{S}_{i}$. Notice that, in general, the shapes of UBCIRs for a given constellation depend on the Voronoi regions, as illustrated in Fig. 1 (green shaded regions with dashed boundary edges).

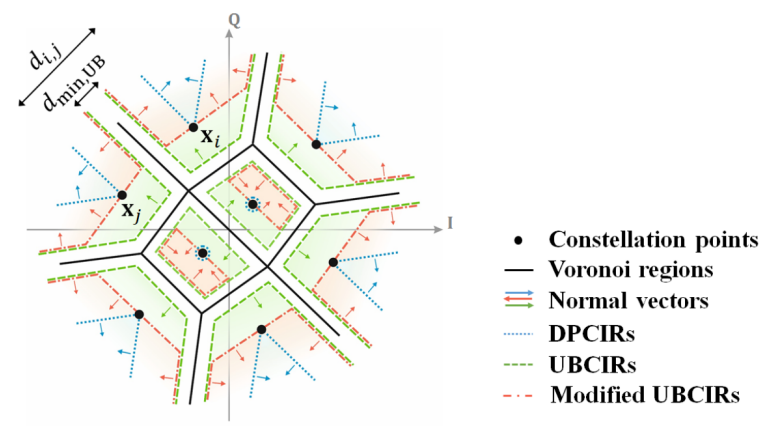

Fig. 1. An illustration of DPCIRs and UBCIRs for the AWGN-optimized 8 -ary constellation. The CIR hyperplanes are depicted by dotted and dashed lines, and shaded areas represent the intersection of halfspaces.

$\mathcal{D}_{i, \mathrm{UB}}$ as defined in (8) may not fulfill the amplitude condition deduced from (1). Therefore, we should consider an additional constraint for each constellation point $\mathbf{x}_{i}$. In fact, the amplitude condition is satisfied if the relaxed CIR associated with $\mathbf{x}_{i}$ is a subset of the complementary region of the disc centered at the origin passing through $\mathbf{x}_{i}$. However, intersecting such a region with $\mathcal{D}_{i, \mathrm{UB}}$ yields a non-convex set. An approximate alternative is to consider the outward halfspace generated by the hyperplane tangent to the disc at $\mathbf{x}_{i}$. This halfspace can be specified by a normal vector $\mathbf{a}_{i, 0}$ parallel to $\mathbf{x}_{i}$ and the offset constant $b_{i, 0}=\mathbf{a}_{i, 0}^{T} \mathbf{x}_{i}$. Subsequently, $\mathbf{A}_{i}$, $\mathbf{b}_{i}$ and $\Delta_{i, \mathrm{UB}}$ in (8) are replaced with $\mathbf{A}_{i}^{\prime}=\left[\mathbf{A}_{i}^{T} \mid \mathbf{a}_{i, 0}\right]^{T}$, $\mathbf{b}_{i}^{\prime}=\left[\mathbf{b}_{i}^{T} \mid b_{i, 0}\right]^{T}, \Delta_{i, \mathrm{UB}}^{\prime}=\left[\Delta_{i, \mathrm{UB}}^{T} \mid 0\right]^{T}$, respectively, where $[\cdot \mid \cdot]$ denotes an augmented vector. Loosely speaking, we also refer to these modified regions as UBCIRs in the following. These regions are red-shaded in Fig. 1 with dash-dotted boundary edges.

The definitions of DPCIR and UBCIR are valid for all generic constellations since they depend only on the Voronoi regions. It is also important to note that one can relax the DPCIRs such that the distance between each CIR hyperplane and the corresponding Voronoi boundary is $d_{\min }$. In this case, the upper bound on SER provided by the NNUB (6) remains unchanged (with respect to the original constellation) as the minimum distance of the constellation is preserved. These relaxed regions may be referred to as minimum distance preserving CIRs (MDPCIR). For the constellation point $\mathbf{x}_{i}$, if there exists at least one neighboring point $\mathbf{x}_{j}$ with $d_{i, j}>d_{\min }$, the associated MDPCIR will be larger than $\mathcal{D}_{i, \mathrm{DP}}$, but not larger than $\mathcal{D}_{i, \mathrm{UB}}$. Therefore, in the rest we only concentrate on DPCIRs and UBCIRs.

\section{Optimization Problem Formulation}

Having DPCIRs and UBCIRs defined, we can formulate the SLP design problem for the generic constellation set $\chi$. In particular, we are interested in power minimization problem with SNR constraints. To this end, we use the hyperplane representation of DPCIRs or UBCIRs to form the set of constraints in the optimization problem (2). If the constraints are defined based on DPCIRs, we call the problem DP-SP or DP-PP, depending on whether sum or peak (per-antenna) transmit power is minimized. Similarly, for UBCIR-based constraints, we call the problem UB-SP or UB-PP. 
Each symbol $s_{k}, k=1, \ldots, K$, corresponds to one of the points $\left\{\mathbf{x}_{i}\right\}_{i=1}^{M}$. We denote by $i_{k}$ the index of the constellation point corresponding to $s_{k}$, thus $i_{k} \in\{1, \ldots, M\}, k=1, \ldots, K$. Using (5), DP-SP and DP-PP problems can be written as

$$
\begin{array}{r}
\mathbf{u}(\mathbf{s}, \mathbf{H}, \gamma)=\underset{\mathbf{u}}{\operatorname{argmin}} f(\mathbf{u}) \\
\text { s.t. } \mathbf{A}_{i_{k}}\left[\begin{array}{l}
\Re\left\{\mathbf{h}_{k} \mathbf{u}\right\} \\
\Im\left\{\mathbf{h}_{k} \mathbf{u}\right\}
\end{array}\right] \succeq \sigma \sqrt{\gamma_{k}}\left(\mathbf{b}_{i_{k}}+\Delta_{i_{k}, \mathrm{DP}}\right), \\
k=1, \ldots, K,
\end{array}
$$

and using (8), UB-SP and UB-PP problems are formulated as

$$
\begin{aligned}
& \mathbf{u}(\mathbf{s}, \mathbf{H}, \boldsymbol{\gamma})=\underset{\mathbf{u}}{\operatorname{argmin}} f(\mathbf{u}) \\
& \text { s.t. } \quad \mathbf{A}_{i_{k}}^{\prime}\left[\begin{array}{r}
\Re\left\{\mathbf{h}_{k} \mathbf{u}\right\} \\
\Im\left\{\mathbf{h}_{k} \mathbf{u}\right\}
\end{array}\right] \succeq \sigma \sqrt{\gamma_{k}} \mathbf{b}_{i_{k}}^{\prime}+\Delta_{i_{k}, \mathrm{UB}}^{\prime}, \\
& k=1, \ldots, K,
\end{aligned}
$$

to find the optimal transmit vector $\mathbf{u}$ with minimum sum, i.e., $f(\mathbf{u})=\|\mathbf{u}\|_{2}^{2}$, or peak per-antenna, i.e., $f(\mathbf{u})=\|\mathbf{u}\|_{\infty}^{2}$, power. Due to convex objective functions and linear inequality constraints, both the optimization problems are convex and can efficiently be solved via standard methods [11]. In general, the number of constraints in DP-SP or DP-PP varies from $2 K$ to $\sum_{k} M_{i_{k}}$, and in UB-SP or UB-PP varies from $3 K$ to $K+\sum_{k} M_{i_{k}}$, depending on the adopted constellation.

\section{Simulation Results}

In this section, we present the simulation results obtained from averaging the multiuser MISO downlink transmission over 100 frames of 50 symbols. The simulations are done using MATLAB software and CVX convex programming package (SDPT3 solver). The fading coefficients are generated every frame and distributed as $\mathbf{H} \sim \mathcal{C N}\left(0, \mathbf{I}_{N}\right)$, where $\mathbf{I}_{N}$ denotes the identity matrix. We consider identical SNR thresholds for all users, i.e., $\gamma_{k}=\gamma, k=1, \ldots, K$. In UB-SP and UB-PP, a fixed target SER $P_{\mathrm{e}}=10^{-3}$ is assumed for all SNR thresholds.

Fig. 2 shows average per-antenna transmit power (sum power divided by $N$ ) and peak per-antenna transmit power obtained from various SLP power minimization problems for two different constellations. The results are compared to CI zero forcing (CIZF), CI sum power minimization (CIPM) [6], and CI peak power minimization (CIPPM) [7] schemes.

For 8-PSK constellation, Fig. 2 (a), the transmit powers with DP-SP and DP-PP are around $2 \mathrm{dBW}$ less than those obtained by CIPM and CIPPM, respectively. It should be also noted that DP-SP and the SLP power minimization problem presented for PSK constellations in [5] are equivalent due to having same CIRs (as mentioned in Subsection III-A). As expected, UBSP and UB-PP with relaxed CIRs are the most energy-efficient SLP schemes both with $1 \mathrm{dBW}$ less transmit power at $\gamma=23$ $\mathrm{dB}$, compared to DP-SP and DP-PP. This reduction of power is achieved in exchange for possibly higher, but upper bounded by $10^{-3}$, SERs. In order to have an estimate of the variance of minimum transmit power due to the random channel matrix $\mathbf{H}$, we have also simulated 700 frames of 100 symbols for 8PSK constellation at $\gamma=21 \mathrm{~dB}$. The results show a maximum of $4 \%$ with respect to those shown in Fig. 2 (a).

Similar trends can be observed in Fig. 2 (b) for the optimal transmit powers obtained from different SLP problems with

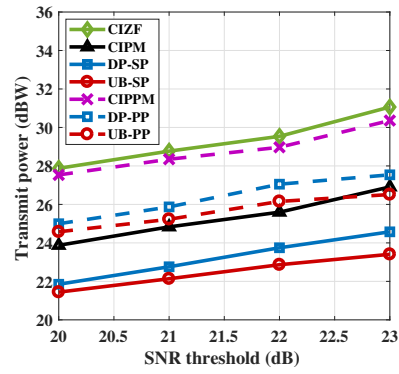

(a) 8-PSK

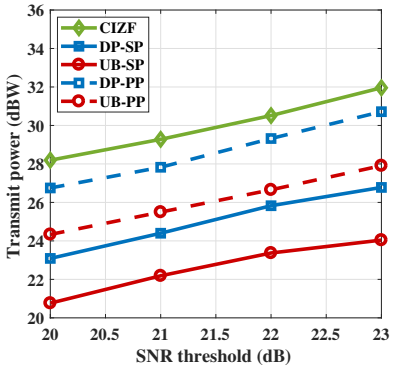

(b) Optimized 8-ary
Fig. 2. Transmit power versus SNR threshold with $N=K=8$ and $\sigma^{2}=1$. The solid and dashed curves show average per-antenna and peak per-antenna transmit powers, respectively.

optimized 8-ary constellation. Notice that CIPM and CIPPM schemes as formulated in [6] and [7] are not applicable to this constellation.

We also compare the computational complexity of various SLP sum power minimization schemes in terms of the average solution time computed by CVX. The relative solution times (normalized by the smallest value in order to have a meaningful comparison) and the number of constraints are reported in Table I. As it can be seen, CIZF has the lowest computational complexity, but it is the least energy-efficient SLP scheme. DPSP and UB-SP problems both require less time than CIPM to be solved, and indeed they are more energy-efficient.

TABLE I

NUMBER OF CONSTRAINTS AND RELATIVE SOLUTION TIME OF VARIOUS SLP SCHEMES WITH 8-PSK CONSTELLATION.

\begin{tabular}{ccccc}
\cline { 2 - 5 } & CIZF & CIPM & DP-SP & UB-SP \\
\hline Number of constraints & $2 K$ & $3 K$ & $2 K$ & $3 K$ \\
Solution time & 1.000 & 1.330 & 1.192 & 1.318 \\
\hline
\end{tabular}

\section{CONCLUSIONS}

In this letter, first we define DPCIRs for the multiuser SLP problem. These regions are optimal when the original SER is not allowed to increase. In a more flexible case, we consider relaxed CIRs and guarantee the target SER using the union bound. This leads to introducing the UBCIRs. We describe both these CIRs based on the hyperplane representation of decision regions for any given constellation shape and size. We then provide a convex formulation for the SLP power minimization problem defined on DPCIRs and UBCIRs. Our simulation results verify that the obtained transmit vectors reduce the power consumption without imposing additional computational complexity on the transmitter compared to the current schemes.

\section{ACKNOWLEDGMENT}

The authors would like to thank Dr. Maha Alodeh for several discussions. The authors are supported by the Luxembourg National Research Fund under CORE Junior project: C16/IS/11332341 Enhanced Signal Space opTImization for satellite comMunication Systems (ESSTIMS). 


\section{REFERENCES}

[1] E. Björnson, M. Bengtsson, and B. Ottersten, "Optimal multiuser transmit beamforming: A difficult problem with a simple solution structure," IEEE Signal Process. Mag., vol. 31, no. 4, pp. 142-148, Jul. 2014.

[2] Y. F. Liu, Y. H. Dai, and Z. Q. Luo, "Coordinated beamforming for MISO interference channel: Complexity analysis and efficient algorithms," IEEE Trans. Signal Process., vol. 59, no. 3, pp. 1142-1157, Mar. 2011.

[3] M. Schubert and H. Boche, "Solution of the multiuser downlink beamforming problem with individual SINR constraints," IEEE Trans. Veh. Technol., vol. 53, no. 1, pp. 18-28, Jan. 2004.

[4] C. Masouros and E. Alsusa, "Soft linear precoding for the downlink of DS/CDMA communication systems," IEEE Trans. Veh. Technol., vol. 59, no. 1, pp. 203-215, Jan. 2010.

[5] C. Masouros and G. Zheng, "Exploiting known interference as green signal power for downlink beamforming optimization," IEEE Trans. Signal Process., vol. 63, no. 14, pp. 3628-3640, Jul. 2015.

[6] M. Alodeh, S. Chatzinotas, and B. Ottersten, "Constructive multiuser interference in symbol level precoding for the MISO downlink channel," IEEE Trans. Signal Process., vol. 63, no. 9, pp. 2239-2252, May 2015.

[7] D. Spano, M. Alodeh, S. Chatzinotas, and B. Ottersten, "Per-antenna power minimization in symbol-level precoding," in IEEE Global Communications Conf. (GLOBECOM), Dec. 2016, pp. 1-6.

[8] A. $\mathrm{Li}$ and $\mathrm{C}$. Masouros, "Exploiting constructive mutual coupling in P2P MIMO by analog-digital phase alignment," IEEE Transactions on Wireless Communications, vol. 16, no. 3, pp. 1948-1962, Mar. 2017.

[9] M. Alodeh, D. Spano, S. Chatzinotas, and B. Ottersten, "Faster-thanNyquist spatiotemporal symbol-level precoding in the downlink of multiuser MISO channels," in IEEE Int. Conf. on Acoustics, Speech and Signal Processing (ICASSP), Mar. 2017, pp. 3779-3783.

[10] N. D. Sidiropoulos, T. N. Davidson, and Z.-Q. Luo, "Transmit beamforming for physical-layer multicasting," IEEE Trans. Signal Process., vol. 54, no. 6, pp. 2239-2251, Jun. 2006.

[11] S. Boyd and L. Vandenberghe, Convex Optimization. Cambridge Univ. Press, 2004

[12] F. Kayhan and G. Montorsi, "Constellation design for memoryless phase noise channels," IEEE Trans. Wirel. Commun., vol. 13, no. 5, pp. 2874 2883, May 2014.

[13] A. Goldsmith, Wireless Communications. Cambridge Univ. Press, 2005. 